\title{
GLOBAL STABILITY FOR THERMAL CONVECTION IN A COUPLE-STRESS FLUID WITH TEMPERATURE AND PRESSURE DEPENDENT VISCOSITY
}

\author{
SUNIL, SHALU CHOUDHARY \\ Department of Mathematics, National Institute of Technology, Hamirpur, (H.P.) - 177 005, India. \\ E-mail: sunilnitham@gmail.com \\ P. K. BHARTI \\ Department of Mathematics, G. S. S. S. Mandal, Kangra, (H.P.) - 176 001, India.
}

\begin{abstract}
We show that the global nonlinear stability threshold for convection in a couple-stress fluid with temperature and pressure dependent viscosity is exactly the same as the linear instability boundary. This optimal result is important because it shows that linearized instability theory has captured completely the physics of the onset of convection. It has also been found that the couplestress fluid is more stable than the ordinary viscous fluid and then the effect of couple-stress parameter $(F)$ and variable dependent viscosity $(\Gamma)$ on the onset of convection is also analyzed.
\end{abstract}

\section{INTRODUCTION}

Convection hydrodynamic stability theory is mainly concerned with the determination of critical values of Rayleigh number, demarcating a region of stability from that of instability. The potentials of the linear theory of stability and of the energy method are complementary to each other in the sense that the linear theory gives conditions under which hydrodynamic systems are definitely unstable. It cannot with certainty conclude stability. On the other hand, energy theory gives conditions under which hydrodynamic systems are definitely stable. It cannot with certainty conclude instability. Suffering from its basic assumptions, the validity of the linearized stability theory becomes questionable. Hence, the nonlinear approach becomes inevitable to investigate the effects of finite disturbances.

The oldest method of nonlinear stability analysis which can deal with finite disturbances is the energy method, originated by Reynolds [1], Orr [2] and then later Serrin [3] and Joseph [4]-[6] reformulated the energy method. Despite the success of this classical energy method in several stability problems, there is some skepticism about its ongoing indiscriminate use. Situations have been encountered, for example, in magnetic Bénard problem Rionero [7], Galdi [8] and in the rotating Bénard problem (Galdi and Straughan [9]), where the classical energy theory did not produce expected results. Rapid improvements of the classical energy theory have been made in recent years (Galdi and Padula [10], Straughan [11]), and the Lyapunov direct method em- 
ployed by Rionero and Mulone [12], Mulone and Rionero [13], Qin and Kaloni [14] appears to have been the most successful one. It is now generally believed that this generalized energy method is definitely superior to the classical energy method. A nonlinear stability analysis of fluids by using generalized energy stability theory has been considered by many authors (Guo et al. [15], Guo and Kalon [16], Straughan and Walker [17], Kaloni and Qiao [18]-[20], Straughan [21], Payne and Straughan [22], Straughan [23]). Recently, Sunil and Mahajan [24]-[29] studied the nonlinear stability analysis for magnetized ferrofluid by using energy method. They found that the nonlinear critical stability magnetic thermal Rayleigh number does not coincide with that of the linear instability analysis, and thus indicates that the subcritical instabilities are possible. However, it has been noted that, in the case of non-ferrofluid, global nonlinear stability Rayleigh number is exactly the same as that for linear instability. More recently, Sunil et al. [30] studied the global stability analysis for thermal convection in a couple-stress fluid.

With the growing importance of non-Newtonian fluid in modern technology and industries, investigations of such fluids are desirable. The presence of small amounts of additives in a lubricant can improve bearing performance by increasing the lubricant viscosity and thus producing an increase in the load capacity. These additives in a lubricant also reduce the coefficient of friction and increase the temperature range in which the bearing can operate. Stokes [31] proposed a simplest theory called the Stokes micro-continuum theory and which could be used for the simulation of the couple-stress fluid. This kind of couple-stress model is intended to take account of the particle-size effects, and it is also very useful in the scientific and engineering applications. At normal operating conditions, the viscosity of an incompressible fluid is assumed to be independent of the pressure. However, it is well known that the viscosity of a fluid can change with pressure, and if the pressure range is significantly large the viscosity can change by several orders of magnitude. Thus, one could consider such liquids as incompressible fluids with pressure dependent viscosities. In his celebrated paper on the response of fluids, Stokes [32] notes that the viscosity of a fluid could depend upon the pressure. However, based on the experiments on the flow of water in canals and normal operating conditions, Stokes suggested that the viscosity could be considered a constant for flows. Stokes is however very careful to delineate the class of flows wherein viscosity might be considered a constant and he also remarks that such an assumption would be invalid under other flow conditions. As early as 1893, Barus [33] proposed an empirical relationship between the viscosity and the pressure, namely

$$
\mu(p)=\mu_{0} \exp \left[\delta\left(p-p_{0}\right)\right] .
$$

Later, Andrade [34] suggested the following expression for the viscosity

$$
\mu(p, \rho, \theta)=A \rho^{1 / 2} \exp \left[\left(p+\rho r^{2}\right) \frac{s}{T}\right],
$$


based on experiments. More recently, Laun [35] modeled the viscosity of polymer melts through

$$
\mu(p, T)=\mu_{0} \exp \left[\delta\left(p-p_{0}\right)-\gamma\left(T-T_{U}\right)\right] .
$$

There have been numerous other experiments by Laun [36] that show that the dependence of the viscosity on the pressure is exponential. Mention must also be made of the work of Martin-Alfonso et al. [37], [38], wherein an intricate relationship between the temperature, viscosity and pressure is provided for bitumen. Ramanaiah [39] applied the couple-stress fluid model to analyze the long slider bearing. Gupta and Sharma [40] also used the couple-stress fluid model to carry out a hydrostatic thrust bearing. Shehawey and Mekheimer [41] applied the couple-stress model to analyze the peristalsis problem for its relative mathematical simplicity. Das [42] proposed the analysis of elastohydrodynamic theory of line contacts. Das [43] studied the slider bearing lubricated with couple-stress fluids in magnetic field and observed that both the values of the maximum load capacity and the corresponding inlet-outlet film ratio depend on couple-stress, magnetic parameters and the shape of bearings. Abdallah and Lotfi [44] proposed an efficient numerical scheme to solve the direct lubrication problem for journal bearing lubricated with couple-stress fluids, which consists of the modified Reynolds equation, the film thickness equation, and the boundary for the pressure field. Hsu et al. [45] studied the short journal bearings lubricated with the non-Newtonian fluid which combined the effects of couple-stresses and surface roughness. It was found that the combined effects of couple-stress and surface roughness can improve the load carrying capacity and decrease the attitude angle and friction parameters. Lahmar [46] also found that the lubricants with couple-stress would increase the load carrying capacity and stability, and decrease the friction factor and the attitude angle. Recently, Rajagopal et al. [47] extended the approximation due to Oberbeck and Boussinesq to the case of a fluid whose viscosity, specific heat and thermal conductivity depend on both the temperature and pressure. When the material parameters depend only on the temperature, the result established by Rajagopal et al. [47] reduces to the classical Oberbeck-Boussinesq approximation. Using this approximation, Rajagopal et al. [48] studied the problem of Rayleigh-Bènard convection and assuming that the viscosity is an analytic function of the temperature and pressure they studied both the linear as well as the nonlinear stability corresponding to the RayleighBènard problem. They showed that the principle of exchange of stabilities holds and that the critical Rayleigh numbers for the linear and nonlinear stability coincide.

The purpose of the present paper is to study the nonlinear stability as well as linear instability analysis of couple-stress fluid with temperature and pressure dependent viscosity heated from below. Here, we establish the optimal result, that is, the linear instability and nonlinear stability Rayleigh numbers are the same. We also find that the critical value of thermal Rayleigh number for the couple-stress fluid is higher than the critical value of thermal Rayleigh number for the ordinary fluid hence the couple- 
stress fluid is more stable than the ordinary fluid. This problem, to the best of our knowledge, has not been investigated yet.

\section{MATHEMATICAL FORMULATION OF THE PROBLEM}

Here, we consider an infinite, horizontal layer of thickness " $d$ " of incompressible thin couple-stress fluid with temperature and pressure dependent viscosity heated from below. The fluid is assumed to occupy the layer $V_{d}=\mathbb{R}^{2} \times(0, d)(d>0)$. The temperature $T$ at the bottom and top surfaces $z=0$ and $d=z$ is $T_{L}, T_{U}$, respectively, and a temperature gradient $\beta\left(=\left|\frac{d T}{d z}\right|\right)$ is maintained. The gravity field $\mathbf{g}=(0,0-g)$ pervades the system in the negative $z$-direction.

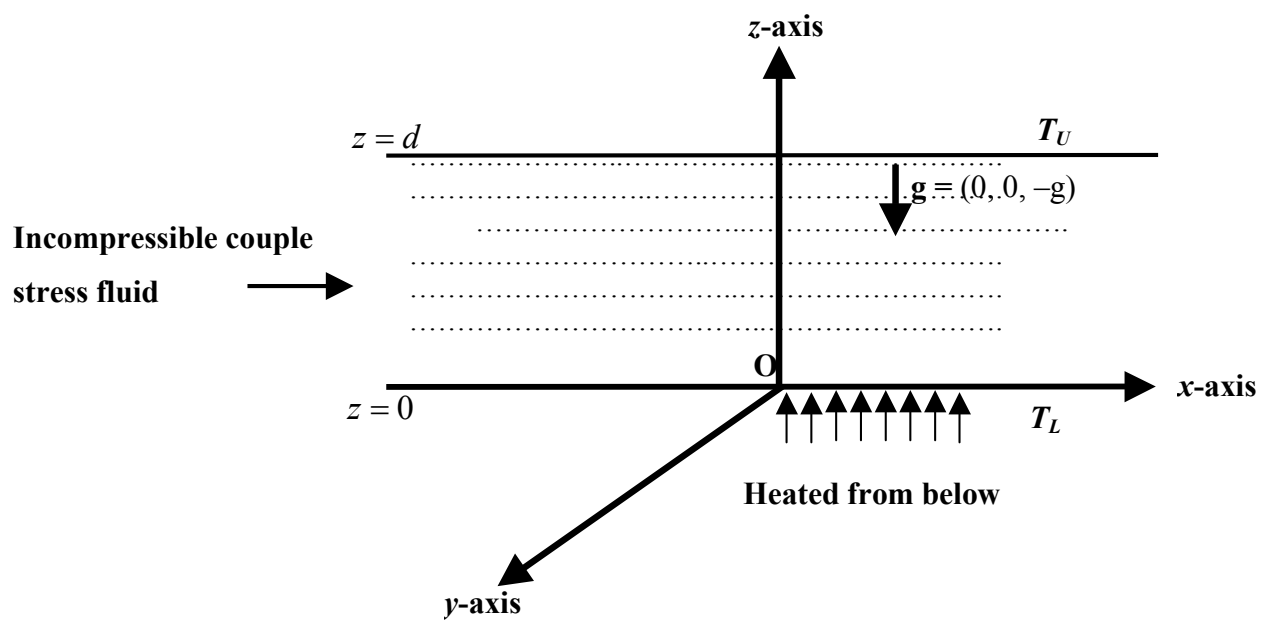

Fig. 1. Geometrical configuration of the problem

The equations governing the flow of an incompressible couple-stress fluid (utilizing the Boussinesq approximation) are given as follows (see Rajagopal et al. [48], Stokes [32])

$$
\begin{gathered}
\nabla p+\rho_{0} g \mathbf{k}=0, \\
\rho_{0}\left(\frac{\partial}{\partial t}+\mathbf{q} \cdot \nabla\right) \mathbf{q}=-\alpha\left(T_{L}-T_{U}\right) \nabla p+\rho_{0} \mathbf{g} \alpha\left(T-T_{U}\right) \mathbf{k} \\
+2[\mathbf{D}] \nabla \mu(p, T)+\left[\mu(p, T)-\mu^{\prime} \nabla^{2}\right] \nabla^{2} \mathbf{q}, \\
\nabla \cdot \mathbf{q}=0,
\end{gathered}
$$




$$
\frac{\partial T}{\partial t}+\mathbf{q} \cdot \nabla T=\kappa \nabla^{2} T .
$$

Here, $\rho, \rho_{0}, \mathbf{q}, \mathbf{g}, t, p, P, \mu, \mu^{\prime}, \kappa$ and $\alpha$ are the fluid density, density at the reference temperature $T_{U}$, velocity, acceleration due to gravity, time, pressure field due to gravity, pressure due to thermal expansion of the fluid, coefficient of viscosity, coefficient of viscoelasticity, thermal diffusivity and coefficient of thermal expansion, respectively, and assume that the $T_{L}$ and $T_{U}$ are the constant temperatures of the lower and upper surfaces of the layer. The appropriate boundary conditions to append to equations (2)-(5) are

$$
T(x, x, 0, t)=T_{L}, \quad T(x, x, d, t)=T_{U}, \quad p(x, x, d, t)=p_{0},
$$

where $p_{0}$ is the reference pressure. Our aim is the study of stability of the steady static conduction solution to equations (2)-(6)

$$
\begin{gathered}
\bar{p}=-\rho_{0} g(z-d)+p_{0}, \\
\overline{\mathbf{q}}=\mathbf{0}, \\
\bar{T}=-\frac{T_{L}-T_{U}}{d} z+T_{L}, \\
\bar{P}=-\rho_{0} g z\left(\frac{z}{2 d}-1\right)+P_{0} .
\end{gathered}
$$

In order to study the stability of the conduction solution, we introduce the perturbations $\mathbf{q}^{\prime}=u \mathbf{i}+v \mathbf{j}+w \mathbf{k}, \theta, p^{\prime}$ and $P^{\prime}$ to $\overline{\mathbf{q}}, \bar{T}, \bar{p}, \bar{P}$, respectively, i.e.,

$$
\mathbf{q}=\overline{\mathbf{q}}+\mathbf{q}^{\prime}, \quad T=\bar{T}+\theta, \quad p=\bar{p}+p^{\prime}, \quad P=\bar{P}+P^{\prime}
$$

The nonlinear equations for the perturbations $\mathbf{q}^{\prime}=(u, v, w), \theta, p^{\prime}, P^{\prime}, \rho_{0}$ which represent velocity, temperature, pressure field due to gravity, pressure due to thermal expansion and density, respectively, are given

$$
\begin{gathered}
\nabla p^{\prime}=0, \\
\rho_{0} \frac{\partial \mathbf{q}^{\prime}}{\partial t}+\rho_{0} \mathbf{q}^{\prime} \cdot \nabla \mathbf{q}^{\prime}=-\alpha\left(T_{L}-T_{U}\right) \nabla P^{\prime}+\mu\left(\bar{p}+p^{\prime}, \bar{T}+\theta\right) \nabla^{2} \mathbf{q}^{\prime} \\
-\mu^{\prime} \nabla^{4} \mathbf{q}^{\prime}+\rho_{0} \alpha g \theta \mathbf{k}+2[\mathbf{D}] \nabla \mu\left(\bar{p}+p^{\prime}, \bar{T}+\theta\right), \\
\nabla \cdot \mathbf{q}^{\prime}=0, \\
\frac{\partial \theta}{\partial t}+\mathbf{q}^{\prime} \cdot \nabla \theta=\kappa \nabla^{2} \theta+\beta w,
\end{gathered}
$$


in $\mathbb{R}^{2} \times(0, d) \times(0, \infty)$ and setting $\mathbf{D}=\left[\nabla \mathbf{q}+(\nabla \mathbf{q})^{T}\right] / 2$. To the previous system, we append the initial conditions

$$
\mathbf{q}(x, 0)=\mathbf{q}_{0}, \quad \theta(x, 0)=\theta_{0}(x),
$$

and the boundary conditions

$$
p^{\prime}(x, y, d, t)=0, \quad \theta=0, \quad u_{z}=v_{z}=w=0 \quad \text { at } \quad z=0, d .
$$

In equation (16), $\mathbf{q}_{0}$ and $\theta_{0}$ are regular fields, $\mathbf{q}_{0}$ being divergence free. From equations (12) and (17) it follows that $p^{\prime} \equiv 0$.

Equations (12)-(15) in non-dimensionalised form (dropping ${ }^{*}$ ) can be written as

$$
\begin{gathered}
\frac{\partial \mathbf{q}}{\partial t}+\mathbf{q} \cdot \nabla \mathbf{q}=-\nabla P+\mu(\bar{p}, \bar{T}+\theta) \nabla^{2} \mathbf{q}-F \nabla^{4} \mathbf{q}+R \theta \mathbf{k}+2[\mathbf{D}] \nabla \mu(\bar{p}, \bar{T}+\theta), \\
\nabla \cdot \mathbf{q}=0, \\
\frac{\partial \theta}{\partial t}+\mathbf{q} \cdot \nabla \theta=\nabla^{2} \theta+R w,
\end{gathered}
$$

with the following non-dimensional quantities and parameters

$$
\left.\begin{array}{c}
t^{*}=\frac{\mu_{0}}{\rho_{0} d^{2}} t, \quad \mathbf{q}^{*}=\frac{\rho_{0} d}{\mu_{0}} \mathbf{q}^{\prime}, \quad P^{*}=\frac{\alpha\left(T_{L}-T_{U}\right) \rho_{0} d^{2}}{\mu_{0}^{2}} P^{\prime}, \quad \theta^{*}=\frac{R}{\beta d} \theta, \quad \mu^{*}=\frac{\mu}{\mu_{0}}, \\
z^{*}=\frac{1}{d} z, \quad \bar{p}^{*}=\frac{\bar{p}-p_{0}}{\rho_{0} g d}=-\left(z^{*}-1\right), \quad \Re=R^{2}=\frac{g \alpha \beta \rho_{0} d^{4}}{\mu_{0} \kappa}, \quad x^{*}=\frac{x}{d}, \\
\bar{T}^{*}=\frac{\bar{T}-T_{U}}{T_{L}-T_{U}}=-\left(z^{*}-1\right), \quad \beta=\frac{T_{L}-T_{U}}{d}, \quad F=\frac{1}{v} \frac{\mu^{\prime}}{\rho_{0} d^{2}} .
\end{array}\right\}
$$

Here, $\mu_{0}=\mu\left(p_{0}, T_{U}\right)$ is the viscosity at the reference state $\left(p_{0}, T_{U}\right), \mathfrak{R}$ is the Rayleigh number and $F$ is the couple-stress parameter.

The functions $\mathbf{q}, \theta$ must be subject to boundary conditions and we suppose that $\mathbf{q}$, $\theta$ are periodic in $x, y$ with periods $\frac{2 \pi}{a_{x}}$ and $\frac{2 \pi}{a_{y}}$, and the surfaces are stress free. Hence, the boundary conditions are given as

$$
u_{z}=0, \quad v_{z}=0, w=0, \quad \theta=0 \text { at } z=0, d .
$$

To exclude the rigid motion we assume that the mean values of $u, v$ are zero (see Kloeden and Well [49]), i.e., we require

$$
\int_{V} u d V=\int_{V} v d V=0
$$


where $V=\left[0, \frac{2 \pi}{a_{x}}\right) \times\left[0, \frac{2 \pi}{a_{y}}\right] \times[0,1]$ is the typical periodicity cell and let $a=\left(a_{x}+a_{y}\right)^{1 / 2}$ be the two dimensional wave number.

\section{NONLINEAR STABILITY ANALYSIS}

Since we have assumed that the viscosity is an analytic function of the temperature and pressure, for sufficiently small disturbances, we can expand the viscosity in the following manner

$$
\mu(\bar{p}, \bar{T}+\theta) \nabla^{2} \mathbf{q}=\left[\sum_{n=0}^{+\infty} \frac{1}{n !} \frac{\partial^{n} \mu}{\partial T^{n}}(\bar{p}, \bar{T}) \theta^{n}\right] \nabla^{2} \mathbf{q} \approx \mu(z) \nabla^{2} \mathbf{q},
$$

and

$$
\begin{gathered}
2 \mathbf{d} \cdot \nabla \mu(\bar{p}, \bar{T}+\theta)=2 \mathbf{d} \cdot\left\{\sum_{n=0}^{\infty} \frac{1}{n !} \nabla\left[\frac{\partial^{n} \mu}{\partial T^{n}}(\bar{p}, \bar{T}) \theta^{n}\right]\right\} \\
\approx \mu^{\prime}\left((z)\left[\left(\frac{\partial u}{\partial y}+\frac{\partial w}{\partial x}\right) \mathbf{i}+\left(\frac{\partial v}{\partial z}+\frac{\partial w}{\partial y}\right) \mathbf{j}+2 \frac{\partial w}{\partial z} \mathbf{k}\right]\right),
\end{gathered}
$$

where

$$
\mu(z)=\mu(\bar{p}, \bar{T}) .
$$

Let $\|\cdot\|$ denote the $L^{2}(V)$ norm. In order to establish a nonlinear stability result, we commence by multiplying equation (18) by $\mathbf{q}$, equation (20) by $\theta$, and we then integrate over $V$ to find

$$
\begin{gathered}
\frac{1}{2} \frac{d}{d t}\|\mathbf{q}\|^{2}=R \int_{V} w \theta d V-F\left\|\nabla^{2} \mathbf{q}\right\|^{2} \\
-\int_{V} \mu(\bar{p}, \bar{T}+\theta) \nabla \mathbf{q} \cdot \nabla \mathbf{q} d V-2 \int_{V} \nabla \mu(\bar{p}, \bar{T}+\theta) \mathbf{d} . \mathbf{d} d V, \\
\frac{1}{2} \frac{d}{d t}\|\theta\|^{2}=R \int_{V} w \theta d V-\|\nabla \theta\|^{2} .
\end{gathered}
$$

Hence, by summing equations (25) and (26), we get 


$$
\begin{aligned}
& \frac{d E}{d t}=\left(1+\lambda_{1}\right) R \int_{V} w \theta d V-\lambda_{1} \int_{V} \mu(\bar{p}, \bar{T}+\theta) \nabla \mathbf{q} \cdot \nabla \mathbf{q} d V \\
& -\lambda_{1} F\left\|\nabla^{2} \mathbf{q}\right\|^{2}-\|\nabla \theta\|^{2}-2 \lambda_{1} \int_{V} \nabla \mu(\bar{p}, \bar{T}+\theta) \mathbf{d} . \mathbf{d} d V,
\end{aligned}
$$

where

$$
E=\frac{1}{2}\|\theta\|^{2}+\frac{\lambda_{1}}{2}\|\mathbf{q}\|^{2}
$$

is the sum of the kinetic and thermal energies associated with the perturbations.

We now state a maximum principle (see Temam [50] page 136 for its proof) which will be very useful for our nonlinear stability analysis.

Lemma. Let the disturbances $\mathbf{q}, P, \theta$ satisfy equations (18)-(20) with boundary conditions

$$
w=\theta=0 \quad \text { at } \quad z=0,1
$$

Then, if

$$
|\theta(x, 0)| \leq \Theta_{0} \quad \forall x \in V,
$$

for constant $\Theta_{0}>0$, it follows that

$$
|\theta(x, t)| \leq \Theta_{0} \quad \forall x \in V \quad \forall t \geq 0 .
$$

As an immediate consequence of Lemma, if the initial disturbance to the temperature field $\bar{T}$ satisfies the inequality

$$
|\theta(x, t)| \leq \Theta_{0} \in \mathbb{R}^{+} \quad \forall x \in V,
$$

by the analyticity of $\mu$, we can write

$$
\mu\left(\bar{p}, \bar{T}+\theta_{0}\right)=\sum_{n=0}^{+\infty} \frac{1}{n !} \frac{\partial^{n} \mu}{\partial T^{n}}(\bar{p}, \bar{T}) \theta_{0}^{n} \quad \forall x \in V,
$$

then

$$
|\theta(x, t)| \leq \Theta_{0} \quad \forall x \in V, \quad \forall t \geq 0,
$$

and further

$$
\mu\left(\bar{p}, \bar{T}+\theta_{0}\right)=\sum_{n=0}^{+\infty} \frac{1}{n !} \frac{\partial^{n} \mu}{\partial T^{n}}(\bar{p}, \bar{T}) \theta_{0}^{n} \quad \forall x \in V, \quad \forall t \geq 0 .
$$

Therefore, for initial thermal disturbances satisfying equation (29), by equations 
(30) and (31), we have

$$
\begin{aligned}
\frac{d E}{d t}= & -\left(1-R \frac{I_{1}}{D_{1}}\right) D_{1}-\lambda_{1} \int_{V}^{+\infty} \frac{1}{n !} \frac{\partial^{n} \mu}{\partial T^{n}}(\bar{p}, \bar{T}+\theta) \theta^{n} \nabla \mathbf{q} \cdot \nabla \mathbf{q} d V-\lambda_{1} \sum_{n-1}^{+\infty} \frac{1}{n !}\left|\frac{\partial^{n} \mu}{\partial T^{n}}\right| \Theta_{0}^{n} \mathbf{d} \cdot \mathbf{d} d V \\
& \leq-\left[1-\frac{R}{R_{E}(a)}\right] D_{1}+\int_{V} \sum_{n=1}^{+\infty} \frac{1}{n !}\left|\frac{\partial^{n} \mu}{\partial T^{n}}\right| \Theta_{0}^{n} \nabla \mathbf{q} \cdot \nabla \mathbf{q} d V+\int_{V} \sum_{n=1}^{+\infty} \frac{1}{n !}\left|\frac{\partial^{n} \mu}{\partial T^{n}}\right| \Theta_{0}^{n} \mathbf{d} \cdot \mathbf{d} d V \\
& \leq-\left[1-\frac{R}{R_{E}(a)}\right] D_{1}+M \lambda_{1}\left(\|\nabla \mathbf{q}\|^{2}+2\|\mathbf{d}\|^{2}\right)
\end{aligned}
$$

where

$$
\begin{gathered}
I_{1}=\left(1+\lambda_{1}\right) R \int_{V} w \theta d V, \\
D_{1}=\lambda_{1} \int_{V} \mu(z) \nabla \mathbf{q} \cdot \nabla \mathbf{q} d V+\lambda_{1} F\left\|\nabla^{2} \mathbf{q}\right\|^{2}+\|\nabla \theta\|^{2}+2 \lambda_{1} \int_{V} \nabla \mu(\bar{p}, \bar{T}+\theta) \mathbf{d} . \mathbf{d} d V, \\
\frac{1}{R_{E}(a)}=\max _{\mathscr{V}} \frac{I_{1}}{D_{1}} .
\end{gathered}
$$

$W$ being the set of the kinematically admissible perturbations

$$
\begin{gathered}
\mathscr{W}=\left\{(\mathbf{q}, \theta) \in\left(H^{1}(V)\right)^{4}: \mathbf{q}, \theta \text { periodic in } x \text { and } y \text { of periods } 2 \pi / a_{x}, 2 \pi / a_{y}\right. \\
\left.\mathbf{q} \text { satisfies }(22), \nabla \cdot \mathbf{q}=0, \frac{\partial u}{\partial z}=\frac{\partial v}{\partial z}=w=\theta=0 \text { at } z=0,1\right\},
\end{gathered}
$$

and

$$
M=\max _{z \in[0,1]} \sum_{n=1}^{+\infty} \frac{1}{n !}\left|\frac{\partial^{n} \mu}{\partial T^{n}}(z)\right| \Theta_{0}^{n} .
$$

The existence of the maximum of the functional $I_{1} / D_{1}$ in the linear space of the kinematically admissible disturbances $W$ can be proved as in Rionero [51].

By assuming

$$
R<R_{E}(a),
$$

and by choosing $\Theta_{0}$, such that 


$$
M<\left[1-\frac{R}{R_{E}(a)}\right] \min _{z \in[0,1]} \mu(z),
$$

from equation (32), by the Poincaré and Wirtinger inequalities, we deduce the following energy inequality

$$
\frac{d E}{d t} \leq-\left[1-\frac{R}{R_{E}(a)}\right] v_{a} E(t),
$$

where

$$
\begin{gathered}
v_{a}=2 \min \left\{\pi_{0}^{2}\left[\min _{z \in[0,1]} \mu(z)-\frac{M R_{E}(a)}{R_{E}(a)-R}\right], \pi^{2}\right\}, \\
\pi_{0}^{2}=\min \left\{a_{x}^{2}, a_{y}^{2}, \pi^{2}\right\} .
\end{gathered}
$$

Integrating equation (34), we have

$$
E(t) \leq E(0) \exp \left\{-\left[1-\frac{R}{R_{E}(a)}\right] v_{a} t\right\} .
$$

The number $R_{E}(a)$ is found from the variational problem equation (33) and the Euler-Lagrange equations corresponding to this are

$$
\begin{gathered}
-\nabla \vartheta=\mu(z) \nabla^{2} \mathbf{q}-F \nabla^{4} \mathbf{q}+\mu^{\prime}\left[\left(\frac{\partial u}{\partial z}+\frac{\partial w}{\partial x}\right) \mathbf{i}+\left(\frac{\partial v}{\partial z}+\frac{\partial w}{\partial y}\right) \mathbf{j}+2 \frac{\partial w}{\partial z} \mathbf{k}\right]+R \theta \mathbf{k}, \\
\nabla \cdot \mathbf{q}=0 \\
\nabla^{2} \theta+R w=0
\end{gathered}
$$

where $\vartheta$ is a Lagrange multiplier associated with the divergence constraint. This eigenvalue problem is exactly the same as the one of linear stability theory and hence the critical Rayleigh numbers for the linear and nonlinear stability problems coincide. Finally, by Lemma and by equation (35), we may state the following:

Theorem 1. Assume that

$$
\mathfrak{R}<\mathfrak{R}_{c}
$$

with $\mathfrak{R}_{c}$ and

$$
\left|\theta_{0}(x)\right| \leq \Theta_{0}, \quad \forall x \in \mathbb{R}^{2} \times[0,1],
$$

for constant $\Theta_{0}>0$, such that 


$$
M=\max _{z \in[0,1]} \sum_{n=1}^{+\infty} \frac{1}{n !}\left|\frac{\partial^{n} \mu}{\partial T^{n}}(z)\right| \Theta_{0}^{n}<\left(1-\sqrt{\frac{\mathfrak{R}}{\mathfrak{R}_{c}}}\right) \min _{z \in[0,1]} \mu(z) .
$$

Then, the conduction solution is nonlinearly stable with respect to the energy of the perturbations $E(t)$, and

$$
E(t) \leq E(0) \exp \left\{-\left[1-\sqrt{\frac{\mathfrak{R}}{\mathfrak{R}_{c}}}\right] v t\right\}
$$

where

$$
v=2 \min \left\{\pi_{0}^{2}\left[\min _{z \in[0,1]} \mu(z)-M\left(1-\sqrt{\frac{\mathfrak{R}}{\mathfrak{R}_{c}}}\right)^{-1}\right], \pi^{2}\right\} .
$$

\section{Variational problem}

We now return to equation (33) and use calculus of variation to find the maximum problem at the critical argument $R_{E}(a)=1$. Using equation (23) and the associated Euler-Lagrange equations after taking transformations $\hat{\mathbf{q}}=\sqrt{\lambda_{1}} \mathbf{q}$ (dropping caps) we have

$$
\begin{aligned}
-2 F \nabla^{4} \mathbf{q}+2 \mu(z) \nabla^{2} \mathbf{q} & +2 \mu^{\prime}(z)\left[\left(\frac{\partial u}{\partial z}+\frac{\partial w}{\partial x}\right) \mathbf{i}+\left(\frac{\partial v}{\partial z}+\frac{\partial w}{\partial y}\right) \mathbf{j}+2 \frac{\partial w}{\partial z} \mathbf{k}\right] \\
& +R\left(1+\lambda_{1}\right) \frac{1}{\lambda_{1}^{1 / 2}} \theta \mathbf{k}=2 \nabla \eta \\
& 2 \nabla^{2} \theta+R\left(1+\lambda_{1}\right) \frac{1}{\lambda_{1}^{1 / 2}} w=0
\end{aligned}
$$

where $\eta$ is the Lagrange multiplier introduced, since $\mathbf{q}$ is solenoidal.

On taking the curl curl of equation (39) and then taking the third component of the resulting equation, we find

$$
\begin{gathered}
4 \mu^{\prime}(z) \frac{\partial \nabla^{2} w}{\partial z}+2 \mu(z) \nabla^{4} w-2 F \nabla^{6} w+2 \mu^{\prime \prime}(z) \frac{\partial^{2} w}{\partial z^{2}}-2 \mu^{\prime \prime}(z) \nabla_{1}^{2} w \\
+\left(1+\lambda_{1}\right) \frac{1}{\lambda_{1}^{1 / 2}} R \nabla_{1}^{2} \theta=0 .
\end{gathered}
$$

Now, we assume a plane tiling form

$$
(w, \theta)=[W(z), \Theta(z)] g(x, y) .
$$


The wave number is found a posteriori to be non-zero, so from equations (41) and (40), we see that $W, \Theta$ satisfy

$$
\begin{gathered}
4 \mu^{\prime}(z) D W\left(D^{2}-a^{2}\right)+2 \mu(z)\left(D^{2}-a^{2}\right)^{2} W-2 F\left(D^{2}-a^{2}\right)^{3} W \\
+2 \mu^{\prime \prime}(z)\left(D^{2}-a^{2}\right) W-R\left(1+\lambda_{1}\right) \frac{1}{\lambda_{1}^{1 / 2}} a^{2} \Theta=0, \\
2\left(D^{2}-a^{2}\right) \Theta+R\left(1+\lambda_{1}\right) \frac{1}{\lambda_{1}^{1 / 2}} W=0,
\end{gathered}
$$

subject to the boundary conditions

$$
W=0, \quad D^{2} W=0, \quad D^{4} W=0, \quad \Theta=0, \quad \text { at } \quad z=0,1 .
$$

\section{NUMERICAL RESULTS}

We consider the pressure-temperature-viscosity relationship equation (1), nondimensionalised as indicated in equation (21), and obtain the dimensionless viscosity

$$
\mu(z)=\exp [\Gamma(z-1)]
$$

with $\Gamma=\gamma\left(T_{L}-T_{U}\right)-\delta \rho_{0} g d$.

Using (46) in equations (43) and (44),

$$
\begin{gathered}
4 \Gamma D\left(D^{2}-a\right)^{2} W+2\left(D^{2}-a^{2}\right)^{2} W+2 \Gamma^{2}\left(D^{2}-a^{2}\right)^{2} W \\
=\exp \left[-\Gamma(z-1)\left(R\left(1+\lambda_{1}\right) \frac{1}{\lambda_{1}^{1 / 2}} a^{2} \Theta+2 F\left(D^{2}-a^{2}\right)^{3} W\right),\right. \\
2\left(D^{2}-a^{2}\right) \Theta+R\left(1+\lambda_{1}\right) \frac{1}{\lambda_{1}^{1 / 2}} W=0,
\end{gathered}
$$

together with boundary conditions equation (45).

By using the Galerkin-type method developed by Chandrasekhar [52], we find approximations to the critical thermal Rayleigh number for different values of the variable dependent viscosity $\Gamma$, couple-stress parameter $F$. For this, we choose $W$ and $\Theta$, satisfying the boundary conditions equation (45) as given by

$$
W=A_{0} \sin \pi z, \quad \Theta=B_{0} \sin \pi z,
$$

where $A_{0}$ and $B_{0}$ are constants. Substituting solution equations (49) in equations (47) and (48), we get the equations involving the coefficients of $A_{0}$ and $B_{0}$. For the existence of non-trivial solutions, the determinant of the coefficients of $A_{0}$ and $B_{0}$ must 
vanish. This determinant on simplification yields

$$
\Re=\frac{\left(\pi^{2}+a^{2}\right)\left[8\left(\pi^{2}+a^{2}\right)^{2}+4 F(2+\Gamma)\left(\pi^{2}+a^{2}\right)^{3}+8 \Gamma^{2}\left(-\pi^{2}+a^{2}\right)\right]}{a^{2}(2+\Gamma)\left(1+\lambda_{1}\right)^{2} \frac{1}{\lambda_{1}}} .
$$

The maximum value of $\lambda_{1}$ is determined by the condition $\frac{d \Re}{d \lambda_{1}}=0$, and is found to be

$$
\lambda_{1}=1 .
$$

Using equation (51) in equation (50), we have

$$
\mathfrak{R}=\frac{\left(\pi^{2}+a^{2}\right)\left[2\left(\pi^{2}+a^{2}\right)^{2}+F(2+\Gamma)\left(\pi^{2}+a^{2}\right)^{3}+2 \Gamma^{2}\left(-\pi^{2}+a^{2}\right)\right]}{a^{2}(2+\Gamma)} .
$$

As a function of $a, \mathfrak{R}$ given by equation (52) attains its minimum when

$$
\begin{gathered}
6 F(2+\Gamma) a^{8}+\left[8+16(2+\Gamma) \pi^{2} F\right] a^{6}+\left[12 \pi^{2}+4 \Gamma^{2}+12(2+\Gamma) F \pi^{4}\right] a^{4} \\
+\left[4 \pi^{4} \Gamma^{2}-4 \pi^{6}-2 \pi^{8}(2+\Gamma) F\right]=0 .
\end{gathered}
$$

The thermal Rayleigh number $\Re$ is minimized with respect to $a$ and we use the Newton-Raphson iterative scheme to obtain the value of critical wave number and the corresponding critical thermal Rayleigh number $\mathfrak{R}_{c}$ (see Table 1).

As regards the nonlinear energy stability analysis, Theorem 1 may be re-stated as follows:

Theorem 2. Assume that

$$
\mathfrak{R}<\mathfrak{R}_{c}
$$

and

$$
\left|\theta_{0}(x)\right| \leq \Theta_{0}<\frac{\sqrt{\mathfrak{R}}}{\gamma\left(T_{L}-T_{U}\right)} \ln \left[1+\left(1-\sqrt{\frac{\mathfrak{R}}{\mathfrak{R}_{c}}}\right) \exp (-|\Gamma|)\right],
$$

for almost every $x \in \mathbb{R}^{2} \times[0,1]$. Then the conduction solution is nonlinearly stable with respect to the energy of the perturbations $E(t)$, and

$$
E(t) \leq E(0) \exp \left\{-\left[1-\sqrt{\frac{\mathfrak{R}}{\mathfrak{R}_{c}}}\right] v t\right\},
$$

where $v=2 \min \left\{\pi_{0}^{2} A, \pi^{2}\right\}$, 


$$
A=\left\{\begin{array}{c}
\exp (-\Gamma)-\frac{\exp \left[\frac{\gamma\left(T_{L}-T_{U}\right)}{\sqrt{\mathfrak{R}}} \Theta_{0}\right]-1}{1-\sqrt{\frac{\mathfrak{R}}{R_{c}}}} \quad \text { if } \Gamma \geq 0, \\
1-\exp (-\Gamma)-\frac{\exp \left[\frac{\gamma\left(T_{L}-T_{U}\right)}{\sqrt{\mathfrak{R}}} \Theta_{0}\right]-1}{1-\sqrt{\frac{\mathfrak{R}}{R_{c}}}} \text { if } \Gamma<0 .
\end{array}\right.
$$

\section{LINEAR STABILITY ANALYSIS}

In order to illustrate the linear instability analysis, the governing equations are obtained from equations (18)-(20) by omitting the nonlinear terms and we arrive at the linearized form

$$
\begin{gathered}
\frac{\partial \mathbf{q}}{\partial t}=-\nabla P+\mu(z) \nabla^{2} \mathbf{q}-F \nabla^{4} \mathbf{q}+\mu^{\prime}\left[\left(\frac{\partial u}{\partial z}+\frac{\partial w}{\partial x}\right) \mathbf{i}+\left(\frac{\partial v}{\partial z}+\frac{\partial w}{\partial y}\right) \mathbf{j}+2 \frac{\partial w}{\partial z} \mathbf{k}\right]+R \theta \mathbf{k}, \\
\nabla \cdot \mathbf{q}=0 \\
\frac{\partial \theta}{\partial t}-R w=\nabla^{2} \theta
\end{gathered}
$$

On taking the vertical component of the curl of equation (54), we eliminate the pressure $P$ and obtain the coupled system in $w$ and $\theta$

$$
\begin{gathered}
\frac{\partial \nabla^{2} w}{\partial t}=2 \mu^{\prime}(z) \frac{\partial \nabla^{2} w}{\partial z}+\mu(z) \nabla^{4} w-F \nabla^{6} w+\mu^{\prime \prime}(z) \frac{\partial^{2} w}{\partial z^{2}}-\mu^{\prime \prime}(z) \nabla_{1}^{2} w+R \nabla_{1}^{2} \theta, \\
\frac{\partial \theta}{\partial t}-R w=\nabla^{2} \theta
\end{gathered}
$$

where

$$
\nabla_{1}^{2}=\frac{\partial^{2}}{\partial x}+\frac{\partial^{2}}{\partial y}
$$

denotes the two dimensional Laplacian. Since the coefficients in equations (57) and (58) depend only on $z$, the equations admit solutions the depend on $x, y$ and $t$ exponentially. We therefore consider solutions of the form 


$$
\begin{aligned}
& w(x, y, z, t)=W(z) \exp \left[i\left(a_{x} x+a_{y} y\right)+c t\right], \\
& \theta(x, y, z, t)=\Theta(z) \exp \left[i\left(a_{x} x+a_{y} y\right)+c t\right],
\end{aligned}
$$

in which it is understood that the real parts of these expressions must be taken into consideration to obtain physical quantities. The wave speed $c$ may be complex, i.e., $c=c_{r}+i c_{i}$, and the expressions equations (59) and (60) thus represent waves which travel in the $x$ and $y$ co-ordinate directions with phase speed $c_{i} /\left(a_{x}^{2}+a_{y}^{2}\right)^{1 / 2}$ and which grow or decay in time given by $\exp \left(c_{r} t\right)$. Such a wave is stable if $c_{r} \leq 0$, unstable if $c_{r}>0$, and naturally stable if $c_{r}=0$.

If we now let $D=\frac{d}{d z}$, then on substituting the expressions equations (59) and (60) into equations (57) and (58), we obtain the following system of ordinary differential equations

$$
\begin{gathered}
c\left(D^{2}-a^{2}\right) W=\mu(z)\left(D^{2}-a^{2}\right)^{2} W+2 \mu^{\prime}(z) D\left(D^{2}-a^{2}\right) W \\
+\mu^{\prime \prime}(z)\left(D^{2}-a^{2}\right) W-F\left(D^{2}-a^{2}\right)^{3} W-R a^{2} \Theta, \\
c \Theta-R W=\left(D^{2}-a^{2}\right) \Theta,
\end{gathered}
$$

to which we add the boundary conditions

$$
W=D^{2} w=D^{4} W=\Theta=0 \quad \text { at } \quad z=0,1 .
$$

Denoting by the superscript ${ }^{*}$ the complex conjugate, multiplying equation (61) by $W^{*}$, equation (62) by $a^{2} \Theta^{*}$, summing and integrating over the interval $[0,1]$, we have

$$
\begin{gathered}
c \int_{0}^{1}\left[|D W|^{2}+a^{2}\left(|W|^{2}+|\theta|^{2}\right)\right] d z=a^{2} R \int_{0}^{1}\left(\Theta W^{*}+W \Theta^{*}\right) d z \\
-a^{2} \int_{0}^{1}\left(|D \Theta|^{2}+a^{2}|\Theta|^{2}\right) d z-F \int_{0}^{1}\left[\left|D^{3} W\right|^{2}+3 a^{2}\left(\left|D^{2} W\right|^{2}+3 a^{4}|D W|^{2}+a^{6}|W|^{2}\right) d z\right. \\
\left.-\left.\int_{0}^{1} \mu(z)\left(\mid D^{2}+a^{2}\right) W\right|^{2}+4 a^{2}|D W|^{2}\right) d z .
\end{gathered}
$$

Thus the right-hand side of equation (64) is real and considering the imaginary part of equation (64), we find

$$
c_{i}=0 \text {. }
$$

Therefore, the linearized equations (54)-(56) satisfy the principle of exchange of stabilities even in the case of couple-stress fluids with pressure and temperature vis- 
cosity. Thus, to find the instability boundary, the lowest value of $\Re$ for which $c>0$, we solve equations (61) and (62) for the smallest eigenvalue $R_{L}(a)$ with $c=0$ (see Chandrasekhar [52]), that is, we find the least eigenvalue $R_{L}(a)$ of the characteristic value problem which gives the neutrally stable states

$$
\begin{gathered}
\mu(z)\left(D^{2}-a\right)^{2} W+2 \mu^{\prime}(z) D\left(D^{2}-a^{2}\right) W \\
+\mu^{\prime \prime}(z)\left(D^{2}-a^{2}\right) W-F\left(D^{2}-a^{2}\right)^{3} W=R a^{2} \Theta, \\
\left(D^{2}-a^{2}\right) \Theta+R W=0,
\end{gathered}
$$

together with boundary conditions as equation (45).

This eigenvalue problem is exactly the same as equations (43) and (44) for nonlinear stability theory with $\lambda_{1}=1$, and hence the critical Rayleigh numbers for the linear and nonlinear stability problems coincide, i.e., the linear instability $\equiv$ the nonlinear stability boundary, and so no sub-critical instabilities are possible. This result is equivalent to the result given by Joseph [4], [5].

The variation of the critical thermal Rayleigh number $\mathfrak{R}_{c}$ with the couple-stress parameter $(F)$ for different values of $\Gamma$

\begin{tabular}{|c|c|c|c|c|c|c|c|c|}
\hline \multirow{2}{*}{$\Gamma$} & \multicolumn{2}{|c|}{$F=0$} & \multicolumn{2}{c|}{$F=0.1$} & \multicolumn{2}{c|}{$F=0.2$} & \multicolumn{2}{c|}{$F=0.3$} \\
\cline { 2 - 8 } & $a_{c}$ & $\mathfrak{R}_{c}$ & $a_{c}$ & $\mathfrak{R}_{c}$ & $a_{c}$ & $\mathfrak{R}_{c}$ & $a_{c}$ & $\mathfrak{R}_{c}$ \\
\hline 0 & 2.2214 & 657.51 & 1.9713 & 1591.11 & 1.9167 & 2507.76 & 1.8924 & 3421.64 \\
0.5 & 2.2057 & 523.01 & 1.9465 & 1452.16 & 1.8991 & 2367.13 & 1.8790 & 3280.19 \\
1 & 2.1570 & 427.99 & 1.9175 & 1351.36 & 1.8804 & 2264.87 & 1.8652 & 3177.28 \\
1.5 & 2.0700 & 354.46 & 1.8849 & 1271.83 & 1.8606 & 2184.23 & 1.8510 & 3096.17 \\
\hline
\end{tabular}

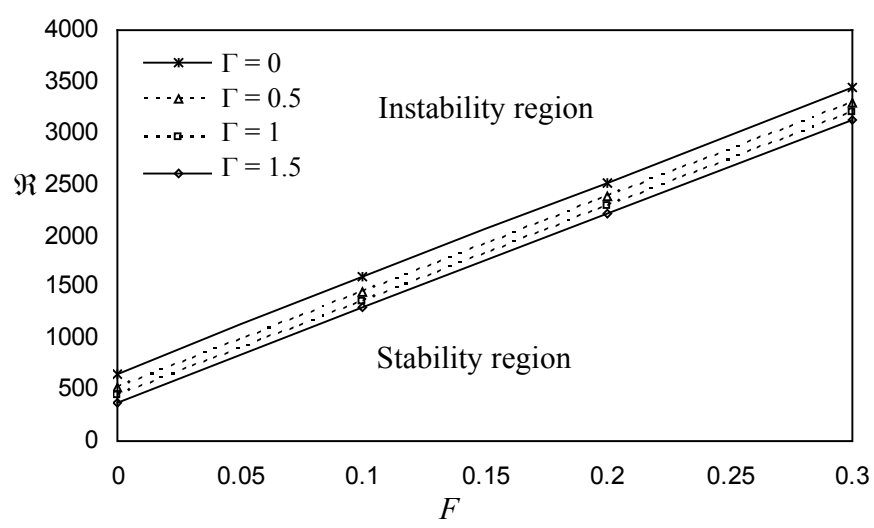

Fig. 2. The variation of the critical thermal Rayleigh number $\Re_{c}$ with the couple-stress parameter $(F)$ for different values of $\Gamma$ 


\section{DISCUSSION OF RESULTS AND CONCLUSIONS}

The critical wave number $a_{c}$ and critical thermal Rayleigh number $\mathfrak{R}_{c}$ depend on the couple-stress parameter $F$ and variable dependent viscosity $\Gamma$. The variation of $\Gamma$, $a_{c}$ and $\Re_{c}$ with variation in $F$ is given in Table 1 and the result is further illustrated in Fig. 2, which represents the plot of critical thermal Rayleigh number $\mathfrak{R}_{c}$ versus the parameter $F$. It is depicted in Fig. 2 that the couple-stress parameter $F$ delays the onset of convection because, as $F$ increases, the value of $\mathfrak{R}_{c}$ increases. Thus, the couplestress parameter has the stabilizing effect on convection. Table 1 indicates that the variable dependent viscosity $\Gamma$ has the destabilizing effect on convection because, as $\Gamma$ increases $(F=0.2)$, the value of $\mathfrak{R}_{c}$ decreases. We observe that for $\Gamma=0$, in particular, for constant dynamic viscosity $(\delta=0=\gamma)$, we obtain classical result. Chandrasekhar [52] also notes that the linear instability and nonlinear stability Rayleigh numbers are both the same and from Table 1, it is clear that for the couple-stress fluid the critical value of Rayleigh number is higher than that of an ordinary fluid.

The principal conclusions from the above analysis are:

1. The result we establish is that the linear instability and nonlinear stability Rayleigh numbers are both the same.

2. This result is equivalent to the result given by Joseph [4], [5] for the standard Bénard problem.

3. The couple-stress has the tendency to slow down the motion of the fluid in the boundary layer, thus reducing the heat transfer from bottom to top. The decrease in heat transfer is responsible for delaying the onset of convection. Thus, the couplestress parameter $F$ promotes stabilization.

4. For the couple-stress fluid the critical value of Rayleigh number is higher than that of an ordinary fluid. Thus, couple-stress fluid is more stable than the ordinary fluid.

5. The variable dependent viscosity $\Gamma$ is found to have destabilizing effect on the system.

\section{REFERENCES}

[1] Reynolds O., Phil. Trans. Roy. Soc. Lond., 1895, A 186, 123.

[2] ORR W. McF., Proc. Roy. Irish. Acad., 1907, A 2, 9.

[3] Serrin J., Arch. Ration. Mech. Analysis, 1959, 3, 1.

[4] JosePH D.D., Arch. Ration. Mech. Analysis, 1965, 20, 59.

[5] JosePh D.D., Arch. Ration. Mech. Analysis, 1966, 22, 163.

[6] Joseph D.D., Stability of Fluid Motions, Springer Verlag, New York, 1976.

[7] Rionero S., Ann. Mat. Pur. Appl., 1968, 78, 339.

[8] GaLdi G.P., Arch. Ration. Mech. Analysis, 1985, 87, 167.

[9] Galdi G.P., Straughan B., Proc. Roy. Soc. Lond., 1985, A 402, 257. 
[10] Galdi G.P., Padula M., Arch. Ration. Mech. Analysis, 1990, 110, 187.

[11] Straughan B., The Energy Method, Stability, and Nonlinear Convection, Springer Verlag, New York, 2004.

[12] Rionero S., Mulone G., Arch. Ration. Mech. Analysis, 1988, 103, 347.

[13] Mulone G., Rionero S., J. Math. Anal. Appl., 1989, 144, 109.

[14] QIN Y., Kaloni P.N., Q. J. Appl. Math., 1995, 53, 129.

[15] Guo J., QIN Y., KaLONI P.N., Int. J. Engg. Sci., 1994, 32, 1207.

[16] Guo J., Kalon P.N., J. Math. Analysis Appl., 1995, 190, 373.

[17] Straughan B., Walker D.W., J. Comput. Phys., 1996, 127, 128.

[18] Kaloni P.N., QiaO Z., Int. J. Heat Mass Transfer, 1997, 40, 1611.

[19] Kaloni P.N., Qiao Z., Int. J. Engg. Sci., 1997, 35, 299.

[20] Kaloni P.N., Qiao Z., Int. J. Heat Mass Transfer, 2001, 44, 1585.

[21] Straughan B., Explosive Instabilities in Mechanics, Springer, Berlin, Germany, 1998.

[22] Payne L.E., Straughan B., Stud. Appl. Math., 2000, 105, 59.

[23] Straughan B., Roy. Soc. Lond., 2001, A 457, 87.

[24] Sunil, Mahajan A., Proc. Roy. Soc. Lond., 2008, A 464, 83.

[25] Sunil, Mahajan A., Appl. Math. Comput., 2008, 204, 299.

[26] Sunil, Mahajan A., Z. Naturforsch., 2008, A 63a, 797.

[27] Sunil, Mahajan A., J. Geophys. Engg., 2008, 5 (3), 311.

[28] Sunil, Mahajan A., Trans. Porous Media, 2009, 76, 327.

[29] Sunil, Mahajan A., ZAMP, 2009, 60, 344.

[30] Sunil, Devi R., Mahajan A., Int. Commun. Heat Mass Transfer, 2011, 38, 938.

[31] StoKes V.K., Phys. Fluids, 1966, 9, 1709.

[32] Stokes G.G., Trans. Camb. Phil. Soc., 1845, 8, 287.

[33] Barus C., Am. J. Sci., 1893, 45, 87.

[34] ANDRADE E.C., Nature, 1930, 125, 309.

[35] LaUn H.M., Rheol. Acta, 2003, 42, 295.

[36] BAir S., KotTKe P., Tribolory Trans., 2003, 46, 289.

[37] Martin-Alfonso M.J., Martinez-Boza F.J., Partal P., Gallegos C., Rheol. Acta, 2006, 45, 357.

[38] Martin-Alfonso M.J., Martinez-Boza F.J., Navarro F.J., Fernandez M., Gallegos C., Fuel, 2007, 86, 227.

[39] RAMANAIAH G., WEAR, 1979, 52, 27.

[40] GuPTA R.S., Sharma L.G., WEAR, 1988, 48, 257.

[41] Shehawey E.F.E., Mekheimer K.S., J. Phys. D. appl. Phys., 1994, 27, 1163.

[42] DAS N.C., STLE Trib. Trans., 1997, 40, 353.

[43] Das N.C., Tribology Int., 1998, 31, 393.

[44] Abdallah A.E., Lotfi H.G., Tribology Int., 2001, 34, 107.

[45] Hsu C.H., Lin J.R. ChiAng H.L., Indus. Lub. Tribology, 2003, 55, 233.

[46] Lahmar M., Proc. Instn. Mech. Engrs., Part J: J. Eng. Tribology, 2005, 219, 145.

[47] Rajagopal K.R., Saccomandi G., Vergori L., Nonlinear Anal. Real Word Appl., 2009, 10 (2), 1139.

[48] Rajagopal K.R., Saccomandi G., Vergori L., ZAMP, 2009, 60, 739.

[49] Kloeden P., Well R., Proc. Roy. Soci. Lond., 1983, A 390, 293.

[50] Temam R., Appl. Math. Sci., 68, Springer-Verlag, New York, Berlin, Heidelberg, 1997.

[51] Rionero S., Annali di Matematica Pura ed Applicata, 1968, 78, 339.

[52] Chandrasekhar S., Hydrodynamic and Hydromagnetic Stability, Dover, New York, 1981. 